Outstanding is the article by Sprent indicating that $T_{H}$ interact only with $B$ cells and macrophages expressing Ia antigens when encountered by the $T$ cells in early differentiation. Confirmatory and contradictory experiments are discussed by Pierce and Kapp, by Mitchison and by Marrack et al. A more detailed analysis of regulatory $\mathrm{T}$ cells requires in vitro experiments. As yet, informative data are rare, but there are some studies in the right direction selection in long-term cultures (Augustin $\boldsymbol{e t}$ $a l$.) and limiting dilution analysis of specific $T_{H}$ (Zauderer et al.), and isolation of a $T_{S}$ clone (Cantor and Gershon) are discussed here. Current experiments concerned with the serology and biochemistry of antigen-specific $\mathrm{T}$ cell receptors and factors are referred to in several articles and some details are described by Cramer and Krawinkel, Goodman et al. and Geha. Melchers and McDevitt present a clearly written, comprehensive review on the selective expression of Ia antigens by $\mathrm{T}$ cells. Functionally distinct human and murine $T$ cell subsets identified by a variety of antisera and monoclonal antibodies feature in papers by Micklem et al., Friedman et al. and Reinherz and Schlossmann; identification of subsets by red cells coated with different classes of antibodies is considered by Fauci and by
Moretta et al. An impressive amount of work, presented or reviewed by Fauci et al., Siegal and Siegal, and Waldmann et al., suggests that a variety of human diseases are associated with or caused by various forms of "disordered immunoregulation".

There is general acceptance of Jerne's notion that antigen-specific receptors must be the primary target of any specific regulatory mechanism in the immune system. This experimentally demanding issue is considered in more detail in articles on idiotype-specific $\mathrm{T}_{\mathrm{S}}$ by Bona and Paul and $T_{H}$ by Eichmann and Simon, $\mathrm{Ig}$ dependent $T_{H}$ by Janeway et al., allotype suppression by Tokuhisa et al. and T cell mediation of resistance to graft versus host disease by Wilson et al. Finally, Herzenberg and colleagues demonstrate how to express complex ideas in clear drawings.

The book will be a disappointment to readers who expect more definitive reports on the various types of $T_{H}$ and $T_{S}, H-2$ restriction of $T_{S}$, inducer cells and feedback inhibition, regulation of cytotoxic $\mathrm{T}$ cell responses or on the various types of $T$ cell factors inducing lymphocyte proliferation and/or differentiation.

Werner Haas is at the Basel Institute for Immunology.

\title{
Unconscious pretension in psychology
}

\section{Stuart Sutherland}

The Psychobiology of Consciousness. Edited by Julian M. Davidson and Richard J. Davidson. Pp.489. ISBN 0-306-40138X. (Plenum: 1980.) \$32.50, £20.48.

ONE of the most significant changes in psychology over the past two decades is that consciousness has become respectable. Several separate developments have brought it back to fashion, including the realization that behaviourism can offer no explanation for many actions, the development of computer simulation as a method for modelling complex thought processes, the increasing interest in Western society in attaining "altered states of consciousness" either through psychotropic drugs or by meditative practices, and the discovery of many different transmitter substances that are involved in the regulation of mood. It is with the latter two themes that The Psychobiology of Consciousness is mainly concerned.

The field appears to be in such chaos that the attempt to think about it has reduced the book's 15 contributors to incoherence. Their strenuous efforts to resuscitate the shade of Titchener by recording and classifying states of consciousness, both normal and abnormal, have met with no success. The few interesting new findings they present have little unity. Typical examples of such isolated discoveries are that since large quantities of marijuana usually have little effect on novices, new users of the drug have to learn how to become "stoned", and that there is a tendency to look to the right (which may mean that the left hemisphere is being used) when thinking verbally, and to the left when working out spatial problems.

Perhaps of more significance are some recent studies of temporal lobe epilepsy. Synchronous spiking of nerve firing within the temporal lobe can be accompanied by feelings of ecstasy, religious awe and extreme kindness, and it is usually associated both with a lack of interest in sex and with the absence of any form of addiction. Treatment of the spike focus to eliminate the hypersynchrony often alters the epileptic's personality for the worse. His irritability increases, and he may become unhappy: he regains an interest in sex, but usually loses his religious preoccupations. Although A.J. Mandel does not go so far as to allege that St Paul had a seizure on the road to Damascus, he does argue that ecstasy is normally accompanied by hypersynchronous discharge in the limbic system excluding the amygdala and that the prolonged state of saintly kindliness that ensues depends upon after-discharges in the same system. There is, however, a danger in generalizing from epileptics to normals since there must be something abnormal in the epileptic brain to cause the discharge in the first place.

In the absence of interesting new results to report, most of the contributors resort to the banal. It is no surprise that the brain waves produced in transcendental meditation resemble those in sleep or that they cannot be distinguished from those occurring when listening to soft music or during progressive relaxation. Nor is it particularly revealing that Italians asked to endure high levels of shock are sensible enough to call off the experiment sooner than do Yankee Protestants.

Much of the theorizing is at the same level of banality. C. E. Izard cites with approval W. Charlesworth's opinion, doubtless reached after years of wrestling with the problem, that "surprise is a misexpected object or event", whilst Karl Pribram, in one of the few intelligible sentences in his chapter, enlightens the ignorant reader with the news that "Science pursues knowledge by observation and experiment".

Most of the book's theorizing is so nonsensical that it is impossible to describe. Its level may be gauged by the following quotation from the chapter by $R$. J. Davidson, one of the editors:

The term 'unconscious' is here being used ... (1) to refer to mental structures... which contour and transform both exogenous (from the environment) as well as endogenous (from within the person....) information, and (2) to refer to processing activity, action and/or information transmission in the nervous system which proceeds in the absence of conscious awareness .... The first usage makes reference to internal mental (i.e. biocognitive) structures The second usage is concerned with observable behaviour (either directly or indirectly) of which the subject is unaware.

One cannot evaluate Dr Davidson's claim to have uncovered two kinds of unconscious process, since he is clearly unable to put the difference into words: nor does he explain what is meant by "observable behaviour of which the subject is unaware". In an equally silly chapter, Pribram argues that "feedforward" operations require consciousness: the reader who wants to know what feedforward is learns that it is "feedbacks joined into parallel processes".

Much of the book - and all of its theoretical sections - is a farrago of pretentious rubbish; it is atrociously written, with the usual attempt to make commonplace ideas sound important by the introduction of neologisms. In short, the book is a disgrace to the publishers, the editors and the contributors.

Stuart Sutherland is Director of the Centre for Research on Perception and Cognition at the University of Sussex. 